\title{
A NON-QUASI-COMPETITIVE COURNOT OLIGOPOLY WITH STABILITY
}

\author{
By Ramon Villanova \\ AND \\ JAUME PARADÍS \\ AND \\ PELEGRÍ Viader
}

Dept. d'Economia i Empresa, Universitat Pompeu Fabra, c/ Ramon Trias Fargas 25-27, 08005-BARCELONA, Spain.

\begin{abstract}
This paper presents a classical Cournot oligopoly model with some peculiar features: it is non-quasi-competitive as price under $N$-poly is greater than monopoly price; Cournot equilibrium exists and is unique with each new entry; the successive equilibria after new entries are stable under the adjustment mechanism that assumes that actual output of each seller is adjusted proportionally to the difference between actual output and profit maximizing output. Moreover, the model tends to perfect competition as $N$ goes to $\infty$, reaching the monopoly price again.
\end{abstract}

KEYworDs: Cournot equilibrium, non-cooperative oligopoly, quasi-competitiveness, stability. 


\section{Introduction}

THE CLASSIC MODEL of Cournot oligopoly equilibrium was designed bearing in mind the mathematical modeling of the "effects of competition". Changing from a monopoly to an oligopoly situation, one of these effects should be, as any reasonable person would agree, the reduction in the price of the commodity. This effect is called quasi-competitiveness in the specialized literature. There exist models in which this effect is not the expected one; in a different scenario to classical Cournot's oligopoly, we may mention Salop (1979), Satterthwaite (1979) and Rosenthal (1980). As for Cournot oligopoly proper, Frank Jr. and Quandt (1963) offer a model in which duopoly price is greater than monopoly price; their inverse demand function is somewhat "kinked" though and the feeling that these kinks are responsible for the rise in price is unavoidable. McManus (1962) and later in McManus (1964) offers more general models in which this situation may happen. In this last paper, McManus (1964), McManus relates quasi-competitiveness with the uniqueness of the equilibrium. Ruffin (1971) presents a classic Cournot equilibrium in which a new entry breaks the quasi-competitiveness, violating at the same time the stability of the model. Ruffin, in fact, directly relates a condition for stability established by Hahn (1962) with quasi-competitiveness. Hahn's condition requires the uniqueness of the equilibrium; Okuguchi and Suzumura (1971) prove that Hahn's stability condition ensures uniqueness of the equilibrium. Lastly, Okuguchi (1974) proves that the uniqueness of Okuguchi and Suzumura proves quasi-competitiveness despite losing stability. ${ }^{1}$ A very good summary of results can be found in Okuguchi (1976) and, from a more general point of view, Daughety (1988).

In this paper, we build a model in which, starting from any linear decreasing inverse demand function and given any number of oligopolists, $N$, we find an increasing piece--wise linear cost function in two pieces such that the model has the following features:

1. Monopoly price, $p_{1}$, is lower than the successive equilibrium prices for duopoly, $p_{2}, 3-$ poly, $p_{3}, \ldots, N$-poly, $p_{N}$. That is to say, $p_{1}<p_{j},(j=$ $2, \ldots, N)$.

2. After each new entry, a unique Cournot equilibrium point is reached.

3. The successive equilibria are stable in a sense that will be seen presently.

\footnotetext{
${ }^{1}$ The models dealt with by these authors vary slightly in their assumptions concerning demand and cost functions: some require differentiability, others only continuity or even semi-continuity. Others consider increasing marginal costs, others not. Some consider all the firms identical and others consider different costs for each firm, etc.
} 
We hope our model may add some information to the clarification of the interdependence of the three aspects of Cournot oligopoly: uniqueness of equilibrium, stability and quasi-competitiveness.

In section 2, after building the model, we discuss monopoly and duopoly maximizing outputs, the reaction curves and the necessary assumptions required to achieve our results. We prove the existence and uniqueness of a Cournot solution and, lastly, we study its stability under an adjustment mechanism proportional with the difference between actual firm output and profit maximizing output. In section 3 we extend the validity of our model to an $N$-poly situation, $N \leq 2$ in which the new firms enter the market one after the other. We prove the existence and uniqueness of the Cournot equilibrium. The stability of the solution for each new entry under the same adjustment mechanism as before is proved in section 4 . Specifically, we prove that for any $N$-poly at the Cournot equilibrium point, if a new firm joins the industry with an output at the most the same as its competitors, the whole $(N+1)$-poly readjust its outputs to reach the new Cournot equilibrium. In section 5 we exhibit a numerical example of our model and, in the conclusions we examine the limiting case of perfect competition as $N \rightarrow \infty$.

\section{The model}

We assume a linear market demand function for the industry of our homogeneous commodity, $p=a-b q, a, b>0$ and a continuous, piece--wise linear cost function:

$$
C(q)= \begin{cases}c_{1}+d_{1} q & \text { if } 0 \leq q<q_{m} \\ c_{2}+d_{2} q & \text { if } q_{m} \leq q \leq a / b\end{cases}
$$

where $c_{1}, c_{2}, d_{1}, d_{2}>0, d_{1} \neq d_{2}$, and $q_{m}$ is a given point in the interval $(0, a / b)$. The continuity of $C(q)$ at $q=q_{m}$ requires that

$$
c_{2}=c_{1}+\left(d_{1}-d_{2}\right) q_{m}
$$

\subsection{Monopoly}

In a monopoly situation, the profit function of our sole firm is:

$$
\Pi(q)= \begin{cases}-b q^{2}+\left(a-d_{1}\right) q-c_{1} & \text { if } 0 \leq q<q_{m} \\ -b q^{2}+\left(a-d_{2}\right) q-c_{2} & \text { if } q_{m} \leq q \leq a / b\end{cases}
$$


that can be written as

$$
\Pi(q)=\left\{\begin{array}{l}
\Pi_{1}(q)=-b\left(q-\frac{a-d_{1}}{2 b}\right)^{2}+\left(\frac{\left(a-d_{1}\right)^{2}}{2^{2} b}-c_{1}\right) \quad \text { if } 0 \leq q<q_{m} \\
\Pi_{2}(q)=-b\left(q-\frac{a-d_{2}}{2 b}\right)^{2}+\left(\frac{\left(a-d_{2}\right)^{2}}{2^{2} b}-c_{2}\right) \quad \text { if } q_{m} \leq q \leq a / b .
\end{array}\right.
$$

The profits are thus denoted separately: $\Pi_{1}(q)$ in the output interval $\left[0, q_{m}\right)$ and $\Pi_{2}(q)$ in the interval $\left[q_{m}, a / b\right]$. The global profit function, $\Pi(q)$, has a derivative for each $q$ in $(0, a / b)$ except for $q=q_{m}$.

Equation (3) represents two parabolas: $\Pi_{1}(q)$ on the left of $q_{m}$ and $\Pi_{2}(q)$ on the right of $q_{m}$. They both connect at $q_{m}$ (see Figure 4 at the end of the paper). By (3) it is obvious that under the assumption $\left(a-d_{1}\right) /(2 b)<q_{m}<$ $\left(a-d_{2}\right) /(2 b)$ the vertex of $\Pi_{1}(q)$ is found at

$$
q_{1}^{c}=\frac{a-d_{1}}{2 b} \quad \text { taking the value } \Pi_{1}^{c}=\frac{\left(a-d_{1}\right)^{2}}{2^{2} b}-c_{1}=b\left(q_{1}^{c}\right)^{2}-c_{1},
$$

and the vertex of $\Pi_{2}(q)$ is at

(5) $q_{2}^{c}=\frac{a-d_{2}}{2 b} \quad$ taking the value $\Pi_{2}^{c}=\frac{\left(a-d_{2}\right)^{2}}{2^{2} b}-c_{2}=b\left(q_{2}^{c}\right)^{2}-c_{2}$.

In order to have $q_{i}^{c}>0,(i=1,2)$, we require that $0<d_{i}<a,(i=1,2)$. Notice that by equations (4) and (5) we get $d_{i}=a-2 b q_{i}^{c}$, and from $0<d_{2}$ we have $q_{2}^{c}<a /(2 b)$. In this way, we impose on our model:

$$
0<q_{1}^{c}<q_{m}<q_{2}^{c}<\frac{a}{2 b}
$$

Introducing the parameters $\lambda=q_{1}^{c} / q_{2}^{c}$ and $\mu=q_{m} / q_{2}^{c}$ the previous expression becomes:

Assumption 1: $\quad 0<\lambda<\mu<1$.

\subsection{Duopoly}

Let us now suppose that a new firm with the same cost function enters the industry. We are now in a situation of duopoly in which $\Pi_{j}$, the profit of firm $j,(j=1,2)$ depends on the output vector $\mathbf{q}=\left(q_{1}, q_{2}\right)$ :

$$
\Pi_{1}(\mathbf{q})= \begin{cases}\Pi_{1,1}=-b q_{1}^{2}+\left(\left(a-d_{1}\right)-b q_{2}\right) q_{1}-c_{1} & \text { if } 0 \leq q_{1}<q_{m} \\ \Pi_{1,2}=-b q_{1}^{2}+\left(\left(a-d_{2}\right)-b q_{2}\right) q_{1}-c_{2} & \text { if } q_{m} \leq q_{1} \leq a / b\end{cases}
$$




$$
\Pi_{2}(\mathbf{q})=\left\{\begin{array}{l}
\Pi_{2,1}=-b q_{2}^{2}+\left(\left(a-d_{1}\right)-b q_{1}\right) q_{2}-c_{1} \quad \text { if } 0 \leq q_{2}<q_{m} \\
\Pi_{2,2}=-b q_{2}^{2}+\left(\left(a-d_{2}\right)-b q_{1}\right) q_{2}-c_{2} \text { if } q_{m} \leq q_{2} \leq a / b .
\end{array}\right.
$$

As above, using (4) and (5), these profit functions can be written as

$\Pi_{1}=\left\{\begin{array}{l}\Pi_{1,1}=-b\left(q_{1}-\left(q_{1}^{c}-\frac{1}{2} q_{2}\right)\right)^{2}+b\left(q_{1}^{c}-\frac{1}{2} q_{2}\right)^{2}-c_{1} \text { if } 0 \leq q_{1}<q_{m} \\ \Pi_{1,2}=-b\left(q_{1}-\left(q_{2}^{c}-\frac{1}{2} q_{2}\right)\right)^{2}+b\left(q_{2}^{c}-\frac{1}{2} q_{2}\right)^{2}-c_{2} \text { if } q_{m} \leq q_{1} \leq a / b\end{array}\right.$

$\Pi_{2}=\left\{\begin{array}{l}\Pi_{2,1}=-b\left(q_{2}-\left(q_{1}^{c}-\frac{1}{2} q_{1}\right)\right)^{2}+b\left(q_{1}^{c}-\frac{1}{2} q_{1}\right)^{2}-c_{1} \quad \text { if } 0 \leq q_{2}<q_{m} \\ \Pi_{2,2}=-b\left(q_{2}-\left(q_{2}^{c}-\frac{1}{2} q_{1}\right)\right)^{2}+b\left(q_{2}^{c}-\frac{1}{2} q_{1}\right)^{2}-c_{2} \quad \text { if } q_{m} \leq q_{2} \leq a / b\end{array}\right.$

\subsection{Reaction curves and reaction functions}

By equation (6), the local maxima of $\Pi_{2}(\mathbf{q})$ in each of the intervals separated by $q_{m}$ are

$$
\Pi_{2}^{\max }=\left\{\begin{array}{l}
b\left(q_{1}^{c}-\frac{1}{2} q_{1}\right)^{2}-c_{1} \\
b\left(q_{2}^{c}-\frac{1}{2} q_{1}\right)^{2}-c_{2}
\end{array}\right.
$$

and these are reached for values of $q_{2}$ that depend on $q_{1}$. This dependence is the reaction curve of firm 2 respect to the output of firm 1:

$$
R_{2}\left(q_{1}\right)= \begin{cases}q_{1}^{c}-\frac{1}{2} q_{1} & \text { if } 0 \leq q_{2}<q_{m} \\ q_{2}^{c}-\frac{1}{2} q_{1} & \text { if } q_{m} \leq q_{2} \leq a / b\end{cases}
$$

In the same way we have the reaction curve of firm 1 respect to the output of firm 2:

$$
R_{1}\left(q_{2}\right)= \begin{cases}q_{1}^{c}-\frac{1}{2} q_{2} & \text { if } 0 \leq q_{1}<q_{m} \\ q_{2}^{c}-\frac{1}{2} q_{2} & \text { if } q_{m} \leq q_{1} \leq a / b\end{cases}
$$

The graph of each reaction curve (see Figure 1) is, in general, the graph of a correspondence and not the graph of a function. $R_{2}\left(q_{1}\right)=q_{m}$ for $q_{1}=2\left(q_{2}^{c}-\right.$ $\left.q_{m}\right)$ and thus, by $(8)$, if $q_{1} \in\left[0,2\left(q_{2}^{c}-q_{m}\right)\right], R_{2}\left(q_{1}\right)$ takes two values. From these two possible values of $R_{2}$, firm 2 will choose the one that maximizes its profit. Using this value as the only image, we will change $R_{2}$ into a proper function.

Consequently, we are interested in finding the $q_{1} \in\left[0,2\left(q_{2}^{c}-q_{m}\right)\right]$ for which we have $\Pi_{2,2}\left(R_{2}\left(q_{1}\right)\right) \geq \Pi_{2,1}\left(R_{2}\left(q_{1}\right)\right)$. By $(7)$ we get:

$$
c_{2}-c_{1} \leq b\left(\left(q_{2}^{c}-\frac{1}{2} q_{1}\right)^{2}-\left(q_{1}^{c}-\frac{1}{2} q_{1}\right)^{2}\right) .
$$


From (2), (4) and (5) we have

$$
c_{2}-c_{1}=2 b\left(q_{2}^{c}-q_{1}^{c}\right) q_{m},
$$

and replacing this value of $c_{2}-c_{1}$ in (9), after some algebra we reach

$$
2 b\left(q_{2}^{c}-q_{1}^{c}\right) q_{m} \leq b\left(q_{2}^{c}+q_{1}^{c}-q_{1}\right)\left(q_{2}^{c}-q_{1}^{c}\right),
$$

which duly simplified takes us to

$$
q_{1} \leq q_{2}^{c}+q_{1}^{c}-2 q_{m} \equiv q_{h}
$$

Notice that $q_{h}<2\left(q_{2}^{c}-q_{m}\right)$. After the correct reaction has been chosen, each

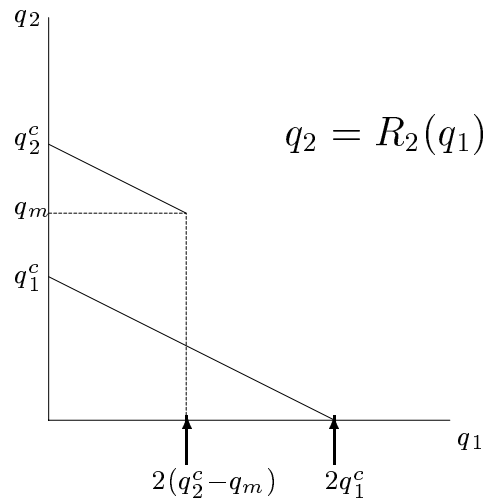

Figure 1

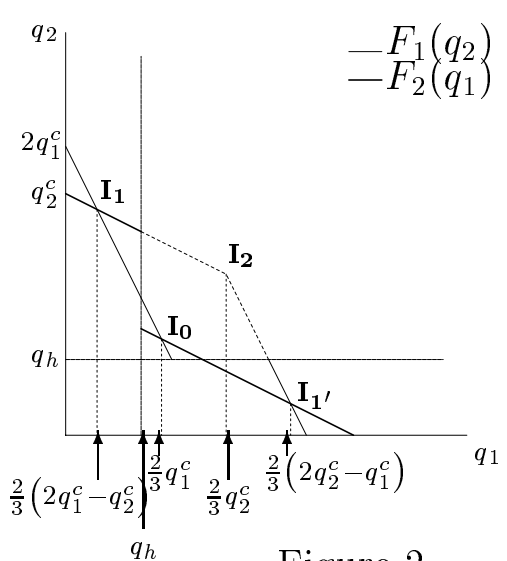

Figure 2

reaction curve becomes a function (see Figure 2),

$$
F_{2}\left(q_{1}\right)= \begin{cases}q_{2}^{c}-\frac{1}{2} q_{1} & \text { if } 0 \leq q_{1} \leq q_{h} \\ q_{1}^{c}-\frac{1}{2} q_{1} & \text { if } q_{h}<q_{1} \leq a / b\end{cases}
$$

and

$$
F_{1}\left(q_{2}\right)= \begin{cases}q_{2}^{c}-\frac{1}{2} q_{2} & \text { if } 0 \leq q_{2} \leq q_{h} \\ q_{1}^{c}-\frac{1}{2} q_{2} & \text { if } q_{h}<q_{2} \leq a / b\end{cases}
$$

The intersections of the reaction functions (11) and (12) are the Cournot points of the model. The possible intersections are

$$
\begin{array}{rlrl}
\mathbf{I}_{\mathbf{0}} & =\left(\frac{2}{3} q_{1}^{c}, \frac{2}{3} q_{1}^{c}\right) ; & \mathbf{I}_{\mathbf{1}}=\left(\frac{2}{3}\left(2 q_{1}^{c}-q_{2}^{c}\right), \frac{2}{3}\left(2 q_{2}^{c}-q_{1}^{c}\right)\right) ; \\
\mathbf{I}_{\mathbf{2}}=\left(\frac{2}{3} q_{2}^{c}, \frac{2}{3} q_{2}^{c}\right) ; & \mathbf{I}_{\mathbf{1}^{\prime}}=\left(\frac{2}{3}\left(2 q_{2}^{c}-q_{1}^{c}\right), \frac{2}{3}\left(2 q_{1}^{c}-q_{2}^{c}\right)\right) .
\end{array}
$$

Not all of these intersections will take place at the same time. Depending on the value of the parameter $q_{h}$ some of them will not be feasible. Let us classify the different possibilities in terms of $q_{h}$ and, consequently, in terms of $q_{m}$. 


\subsection{Conditions for the existence of Cournot points}

For stability reasons, which will be explained in subsection 2.6, we will impose that $q_{2}^{c}<2 q_{1}^{c}$. Recalling that $\lambda=q_{1}^{c} / q_{2}^{c}$, the previous imposition may be written as:

Assumption 2: $\quad \lambda>\frac{1}{2}$.

The function $F_{2}\left(q_{1}\right)$ presents a single discontinuity at $q_{h}=q_{2}^{c}+q_{1}^{c}-2 q_{m}$. From Figure 2 and from the possible positions of $q_{h}$, we infer:

- If $\frac{2}{3}\left(2 q_{2}^{c}-q_{1}^{c}\right) \leq q_{h} \leq q_{2}^{c}$ or, equivalently, $\frac{1}{2} q_{1}^{c} \leq q_{m} \leq \frac{1}{6}\left(-q_{2}^{c}+5 q_{1}^{c}\right)$, the only feasible intersection is $\mathbf{I}_{\mathbf{2}}$.

- If $\frac{2}{3} q_{2}^{c} \leq q_{h}<\frac{2}{3}\left(2 q_{2}^{c}-q_{1}^{c}\right)$ or, equivalently, $\frac{1}{6}\left(-q_{2}^{c}+5 q_{1}^{c}\right)<q_{m} \leq$ $\frac{1}{2}\left(\frac{1}{3} q_{2}^{c}+q_{1}^{c}\right)$, then $\mathbf{I}_{\mathbf{2}}, \mathbf{I}_{\mathbf{1}}$ i $\mathbf{I}_{\mathbf{1}^{\prime}}$ are feasible intersections.

- If $\frac{2}{3} q_{1}^{c} \leq q_{h}<\frac{2}{3} q_{2}^{c}$ or, equivalently, $\frac{1}{2}\left(\frac{1}{3} q_{2}^{c}+q_{1}^{c}\right)<q_{m} \leq \frac{1}{2}\left(q_{2}^{c}+\frac{1}{3} q_{1}^{c}\right)$, then $\mathbf{I}_{\mathbf{1}}$ and $\mathbf{I}_{\mathbf{1}^{\prime}}$ are feasible.

- If $\frac{2}{3}\left(2 q_{1}^{c}-q_{2}^{c}\right) \leq q_{h}<\frac{2}{3} q_{1}^{c}$ or, equivalently, $\frac{1}{2}\left(q_{2}^{c}+\frac{1}{3} q_{1}^{c}\right)<q_{m} \leq \frac{1}{6}\left(5 q_{2}^{c}-q_{1}^{c}\right)$, then we have $\mathbf{I}_{\mathbf{0}}, \mathbf{I}_{\mathbf{1}}$ i $\mathbf{I}_{\mathbf{1}^{\prime}}$ as feasible points.

- Lastly, if $0<q_{h}<\frac{2}{3}\left(2 q_{1}^{c}-q_{2}^{c}\right)$, or, equivalently, $\frac{1}{6}\left(5 q_{2}^{c}-q_{1}^{c}\right)<q_{m}<$ $\frac{1}{2}\left(q_{1}^{c}+q_{2}^{c}\right)$, we will only have $\mathbf{I}_{\mathbf{0}}$.

\subsection{Duopoly vs. monopoly}

Let us turn now to the case we are interested in, the case in which the optima outputs $^{2}$ make the duopoly non-quasi-competitive as $p_{1}<p_{2}$. In order to have this situation, we need that the total industry output under duopoly, $Q_{d}$, be less than the total output under monopoly, $Q_{m}$. This last one can only be $Q_{m}=q_{2}^{c}$ or $Q_{m}=q_{1}^{c}$, whereas the possible values for $Q_{d}$ are:

$$
Q_{d}= \begin{cases}\frac{4}{3} q_{2}^{c} & \text { at } \mathbf{I}_{\mathbf{2}} \\ \frac{2}{3}\left(q_{1}^{c}+q_{2}^{c}\right) & \text { at } \mathbf{I}_{\mathbf{1}} \text { and } \mathbf{I}_{\mathbf{1}^{\prime}} \\ \frac{4}{3} q_{1}^{c} & \text { at } \mathbf{I}_{\mathbf{0}} .\end{cases}
$$

\footnotetext{
${ }^{2}$ In monopoly, the optimum output will be the profit maximizing output. In duopoly, the optima outputs are, ex ante, those of the Cournot equilibrium points: $\mathbf{I}_{\mathbf{0}}, \mathbf{I}_{\mathbf{2}}, \mathbf{I}_{\mathbf{1}}$ and $\mathbf{I}_{1^{\prime}}$.
} 
From $q_{1}^{c}<q_{2}^{c}$ we have the following inequalities

$$
q_{1}^{c}<\frac{4}{3} q_{1}^{c}<\frac{2}{3}\left(q_{1}^{c}+q_{2}^{c}\right)<\frac{4}{3} q_{2}^{c}
$$

and from our second assumption, $q_{2}^{c}<2 q_{1}^{c}$ we infer

$$
q_{2}^{c}<\frac{2}{3}\left(q_{1}^{c}+q_{2}^{c}\right)
$$

Consequently, the only chance of having $Q_{d}<Q_{m}$ is to have a unique Cournot equilibrium point at $\mathbf{I}_{\mathbf{0}}$. In this case, necessarily, $Q_{d}=\frac{4}{3} q_{1}^{c}$ and $Q_{m}=q_{2}^{c}$. We have $\frac{4}{3} q_{1}^{c}<q_{2}^{c}$, which, in terms of our parameter $\lambda$ becomes the third assumption of our model:

Assumption 3: $\quad \lambda<\frac{3}{4}$.

To guarantee the requirement that $Q_{m}=q_{2}^{c}$, monopoly profit for output $q_{2}^{c}$ has to be greater than for output $q_{1}^{c}$. Using (4) and (5), that means

$$
b\left(q_{1}^{c}\right)^{2}-c_{1}<b\left(q_{2}^{c}\right)^{2}-c_{2}
$$

which combined with equation (10) leads to

$$
2 b\left(q_{2}^{c}-q_{1}^{c}\right) q_{m}<b\left(q_{1}^{c}+q_{2}^{c}\right)\left(q_{2}^{c}-q_{1}^{c}\right),
$$

or, simplifying,

$$
q_{m}<\frac{1}{2}\left(q_{1}^{c}+q_{2}^{c}\right)
$$

Recalling that $\mu=q_{m} / q_{2}^{c}$, this last inequality becomes:

Assumption 4: $\quad \mu<\frac{1}{2}(1+\lambda)$.

Now, we have just seen that in order to have $Q_{d}<Q_{m}$ we require that a unique Cournot equilibrium point exists at $\mathbf{I}_{\mathbf{0}}$. From subsection 2.4 this requirement is guaranteed if, and only if,

$$
\frac{1}{6}\left(5 q_{2}^{c}-q_{1}^{c}\right)<q_{m}<\frac{1}{2}\left(q_{1}^{c}+q_{2}^{c}\right),
$$

that, using our parameters $\lambda$ and $\mu$, can be written as:

Assumption 5: $\quad \frac{1}{6}(5-\lambda)<\mu<\frac{1}{2}(1+\lambda)$.

It is worth noticing that by the double inequality in Assumption 5, the parameter $\mu$ has room to exist only if $\lambda>1 / 2$, which is precisely our Assumption 2. Moreover, the second inequality in Assumption5 is exactly Assumption 4. 
It is also clear that Assumption 3, which is needed to ensure $Q_{m}=q_{2}^{c}$, makes $q_{h}>0$ as can be seen from Figure 2. This last condition, makes it possible for firm 2 to maximize its output at $q_{2}=q_{2}^{c}$ if $q_{1}=0$.

Assumptions 1, 2, 3, 4, and 5 can be summarized in the following two double inequalities:

$$
\left\{\begin{aligned}
\frac{1}{2} & <\lambda<\frac{3}{4} \\
\max \left(\lambda, \frac{1}{6}(5-\lambda)\right) & <\mu<\frac{1}{2}(1+\lambda) .
\end{aligned}\right.
$$

\subsection{Stability of the model under duopoly}

We will study the stability of our duopoly model assuming that each firm adjusts its output proportionally with the difference between its actual profit and its profit maximizing output, a common adjustment system in the literature, see Hahn (1962); Fisher (1961); Quandt (1967). We consider that the second firm enters the market when firm 1 is already maximizing its profit producing $q_{2}^{c}$. We will prove that, under the assumptions of our model, the Cournot equilibrium point $\mathbf{I}_{\mathbf{0}}$ is stable no matter the entry output of firm 2 within the range $\left[0, q_{2}^{c}\right]$. We will see that Assumption 2 is essential for the stability of the process as allowing $q_{2}^{c}>2 q_{1}^{c}$ breaks the stability for some productions entries $q_{2}(0) \in\left[0, q_{2}^{c}\right]$ See (16) farther down.

The adjustment mechanism which we consider is

$$
\left\{\begin{array}{l}
\dot{q}_{1}=k_{1}\left(F_{1}\left(q_{2}\right)-q_{1}\right) \\
\dot{q}_{2}=k_{2}\left(F_{2}\left(q_{1}\right)-q_{2}\right),
\end{array}\right.
$$

where $k_{1}$ and $k_{2}$ are the 'speeds' of adjustment, $k_{1}, k_{2}>0$, and $t=0$ is the moment firm 2 enters the market. If we consider as possible outputs all the

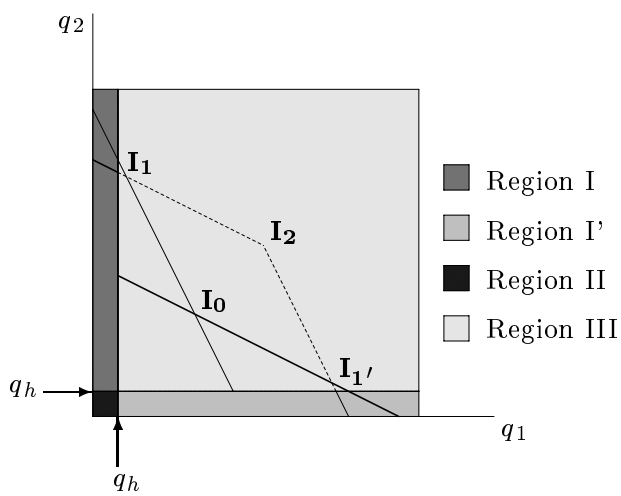

Figure 3. Stability study: The four regions 
points $\left(q_{1}, q_{2}\right) \in[0, a / b] \times[0, a / b]$, the reaction of each firm will depend on the position of the actual outputs $\left(q_{1}(t), q_{2}(t)\right)$ in each of the four regions we can divide the square $[0, a / b] \times[0, a / b]$. Figure 3 shows these four regions which we will call I, I', II and III according to the following description:

- Region I: $0 \leq q_{1} \leq q_{h}$ and $q_{h}<q_{2} \leq a / b$.

- Region I': $q_{h}<q_{1} \leq a / b$ and $0 \leq q_{2} \leq q_{h}$.

- Region II: $0 \leq q_{1} \leq q_{h}$ and $0 \leq q_{2} \leq q_{h}$.

- Region III: $q_{h}<q_{1} \leq a / b$ and $q_{h}<q_{2} \leq a / b$.

In this way, the system of differential equations (14) can be split in four systems, one for each region above, in which the corresponding reaction functions are continuous and linear. To cover these four possibilities, we introduce $A_{1}$ and $A_{2}$ to denote either $q_{1}^{c}$ or $q_{2}^{c}$ depending on the region we are in. Thus, in Region I, $A_{1}=q_{1}^{c}, A_{2}=q_{2}^{c}$; in Region I', $A_{1}=q_{2}^{c}, A_{2}=q_{1}^{c}$; in Region II, $A_{1}=q_{2}^{c}, A_{2}=q_{2}^{c}$ and, lastly, in Region III, $A_{1}=q_{1}^{c}, A_{2}=q_{1}^{c}$. Using this convention, each of the four linear systems in (14) can be written as

$$
\left\{\begin{array}{l}
\dot{q}_{1}=k_{1}\left(A_{1}-\frac{1}{2} q_{2}-q_{1}\right) \\
\dot{q}_{2}=k_{2}\left(A_{2}-\frac{1}{2} q_{1}-q_{2}\right) .
\end{array}\right.
$$

The general solution is:

$$
\left\{\begin{array}{l}
q_{1}(t)=D_{1} e^{-\alpha_{1} t}+E_{1} e^{-\alpha_{2} t}+\frac{2}{3}\left(2 A_{1}-A_{2}\right) \\
q_{2}(t)=D_{2} e^{-\alpha_{1} t}+E_{2} e^{-\alpha_{2} t}+\frac{2}{3}\left(2 A_{2}-A_{1}\right),
\end{array}\right.
$$

where $\alpha_{1}$ and $\alpha_{2}$ are real and positive

$$
\begin{aligned}
& \alpha_{1}=\frac{\left(k_{1}+k_{2}\right)-\sqrt{\left(k_{1}+k_{2}\right)^{2}-3 k_{1} k_{2}}}{2} ; \\
& \alpha_{2}=\frac{\left(k_{1}+k_{2}\right)+\sqrt{\left(k_{1}+k_{2}\right)^{2}-3 k_{1} k_{2}}}{2}
\end{aligned}
$$

and $D_{1}, E_{1}, D_{2}$ i $E_{2}$ real constants that depend on $k_{1}, k_{2}$, and the initial conditions $q_{1}(0)$ and $q_{2}(0)$. The stationary solution is obviously

$$
\left(\frac{2}{3}\left(2 A_{1}-A_{2}\right), \frac{2}{3}\left(2 A_{2}-A_{1}\right)\right),
$$


that is to say, each of the four points $\mathbf{I}_{\mathbf{0}}, \mathbf{I}_{\mathbf{1}}, \mathbf{I}_{\mathbf{1}^{\prime}}, \mathbf{I}_{\mathbf{2}}$, depending on the region in which the motion starts. ${ }^{3}$

The fact that $\alpha_{1}$ and $\alpha_{2}$ in (15) are strictly positive ensures the global stability of each of the attractors if the motion of $\left(q_{1}(t), q_{2}(t)\right)$ stays within the region of validity of the system of differential equations. If the motion of our output vector takes it from one region to another, the values of $A_{1}$ and $A_{2}$ change and the stationary solution with them. It is then required a more detailed study of the motion of our output vector $\left(q_{1}(t), q_{2}(t)\right)$.

If $q_{2}(0)>q_{h}$, the initial point $\left(q_{1}(0), q_{2}(0)\right)$ is located in Region III where $A_{1}=q_{1}^{c}, A_{2}=q_{1}^{c}$ and the attractor is $\mathbf{I}_{\mathbf{0}}$. If $q_{2}(0) \leq q_{h}$, then $\left(q_{1}(0), q_{2}(0)\right)$ is located in Region I' but the corresponding attractor is also placed in Region III and the orbit of $\left(q_{1}(t), q_{2}(t)\right)$ eventually will enter Region III. When this happens, the system of prevailing equations will be the one whose attractor is $\mathbf{I}_{\mathbf{0}}$. The actual orbits may enter or leave different regions depending on the sign of $\dot{q}_{i}$. To be more specific, if $\left(2 q_{1}^{c}-q_{2}^{c}\right) / 2<q_{h}<2\left(q_{1}^{c}-q_{2}^{c}\right) / 3$ there would be orbits going from Region III into Region I' through a point on the segment

$$
\left\{\begin{array}{l}
q_{1}(t) \in\left(2 q_{1}^{c}-2, q_{2}^{c}\right) \\
q_{2}(t)=q_{h}
\end{array}\right.
$$

It is easy to see that, in this case, the second component of the orbit, $q_{2}(t)$, must attain a minimum value exactly on the line $q_{2}=q_{2}^{c}-(1 / 2) q_{1}$, and then will re-enter Region III through a point on the segment

$$
\left\{\begin{array}{l}
q_{1}(t) \in\left(q_{2}^{c}-\frac{1}{2} q_{h}, 2 q_{1}^{c}-2 q_{h}\right) \\
q_{2}(t)=q_{h}
\end{array}\right.
$$

After that, the orbit does not enter Region I' again. A similar behavior may occur when we start from an initial point $q_{2}(0)$ very near to $q_{2}^{c}$ and a value for $k_{1} \gg k_{2}$. In this case, the orbit could move from Region III to Region I behaving in a similar way as before.

To complete the stability study, let us mention that in the case $q_{2}^{c} \geq$ $2 q_{1}^{c}$, proceeding as we did in subsection 2.3 , we would have as one of the intersections

$$
\mathbf{I}_{\mathbf{1}^{\prime}}=\left(\frac{2}{3}\left|2 q_{2}^{c}-q_{1}^{c}\right|,-\frac{2}{3}\left|q_{2}^{c}-2 q_{1}^{c}\right|\right) .
$$

Consequently, in this case, the attractor of an initial state in Region I' would be placed in the non-positive zone for $q_{2}$. That would mean that the output of firm $2, q_{2}(t)$, would decrease till become zero. Firm 2 would leave the market and $\mathbf{I}_{\mathbf{0}}$ would not be stable.

\footnotetext{
${ }^{3}$ Let us recall that we are under the assumptions of our model as summarized in (13). That means that $q_{2}^{c}<2 q_{1}^{c}$, and that the Cournot point $\mathbf{I}_{\mathbf{0}}$ is unique.
} 


\section{Oligopoly}

Let us extend the validity of our model allowing the entry of new firms in the market. We must see how our assumptions concerning $\lambda$ and $\mu$ need be modified as new firms enter the industry, one at a time, with the same cost function, (1), till we get an $N$-poly. We still want to have an increase in the price with respect to the monopoly price, $p_{1}<p_{j},(j=2, \ldots, N)$.

\subsection{Reaction curves and reaction functions}

The profit function of firm $j,(j=1, \ldots, N)$ will depend on the output vector $\mathbf{q}=\left(q_{1}, \ldots, q_{j}, \ldots, q_{N}\right):^{4}$

$$
\begin{aligned}
& \Pi_{j}(\mathbf{q})=
\end{aligned}
$$

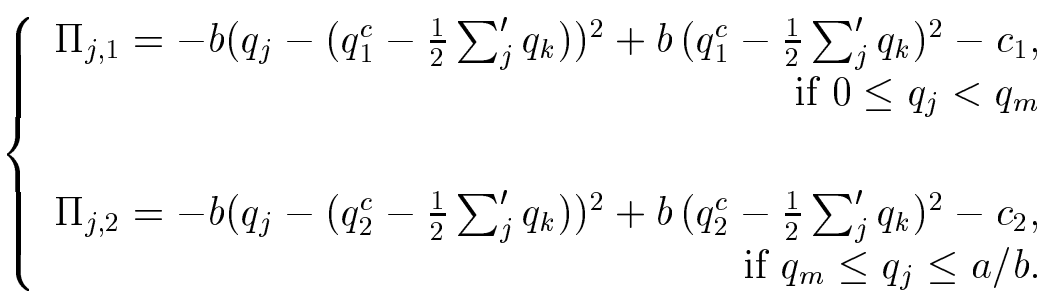

The reactions curve of firm $j$ with respect to its competitors is:

$$
R_{j}\left(q_{1}, \ldots, q_{j-1}, q_{j+1}, \ldots, q_{N}\right)= \begin{cases}q_{1}^{c}-\frac{1}{2} \sum_{j}^{\prime} q_{k} & \text { if } q_{j}<q_{m} \\ q_{2}^{c}-\frac{1}{2} \sum_{j}^{\prime} q_{k} & \text { if } q_{m} \leq q_{j} \leq a / b .\end{cases}
$$

As in duopoly, we notice that for $0 \leq \sum_{j}^{\prime} q_{k} \leq 2\left(q_{2}^{c}-q_{m}\right), R_{j}$ takes two values. Once more, we are interested in finding the $\sum_{j}^{\prime} q_{k}$ for which we have $\Pi_{j, 2}\left(R_{j}\right) \geq \Pi_{j, 1}\left(R_{j}\right)$. Using equation (17) and $c_{2}-c_{1}=2 b\left(q_{2}^{c}-q_{1}^{c}\right) q_{m}$ we have that this is true if

$$
\sum_{j}^{\prime} q_{k} \leq q_{1}^{c}+q_{2}^{c}-2 q_{m}=q_{h}
$$

With this we get the reaction as a true function:

$$
F_{j}\left(q_{1}, \ldots, q_{j-1}, q_{j+1}, \ldots, q_{N}\right)= \begin{cases}q_{2}^{c}-\frac{1}{2} \sum_{j}^{\prime} q_{k} & \text { if } \sum_{j}^{\prime} q_{k} \leq q_{h} \\ q_{1}^{c}-\frac{1}{2} \sum_{j}^{\prime} q_{k} & \text { if } q_{h}<\sum_{j}^{\prime} q_{k} \leq a / b .\end{cases}
$$

\footnotetext{
${ }^{4}$ We use the notation $\sum_{j}^{\prime} q_{i}$ to denote total output of all the industry excluding firm $j$ : $\sum_{j}^{\prime} q_{i}=\left(q_{1}+\cdots+q_{N}\right)-q_{j}$.
} 
As we have two choices for each function, there exist $2^{N}$ intersections, and consequently, $2^{N}$ possible Cournot points. If, as before, we denote $A_{j}$ as one of the possible values $q_{1}^{c}$ or $q_{2}^{c}$, we can write the corresponding system as:

$$
\left\{\begin{array}{l}
q_{1}=A_{1}-\frac{1}{2}\left(q_{2}+q_{3}+\cdots+q_{j}+\cdots+q_{N}\right) \\
\vdots \\
q_{j}=A_{j}-\frac{1}{2}\left(q_{1}+q_{2}+\cdots+q_{j-1}+q_{j+1}+\cdots+q_{N}\right) \\
\vdots \\
q_{N}=A_{N}-\frac{1}{2}\left(q_{1}+q_{2}+\cdots+q_{j}+\cdots+q_{N-1}\right) .
\end{array}\right.
$$

Its solution ${ }^{5}$ is

$$
\left(2 A_{1}-\frac{2 \sum_{k=1}^{N} A_{k}}{N+1}, \ldots, 2 A_{j}-\frac{2 \sum_{k=1}^{N} A_{k}}{N+1}, \ldots, 2 A_{N}-\frac{2 \sum_{k=1}^{N} A_{k}}{N+1}\right) .
$$

The industry output at each of these points is $Q(N)=2 \sum_{k=1}^{N} A_{k} /(N+1)$.

\subsection{N-poly vs. monopoly}

If for any number of firms in the oligopoly, $N \geq 2$, the price $p_{N}$ has to be greater than the price of monopoly, $p_{1}$, and, consequently, $\sum q_{i}<q_{2}^{c}$ we need to have

$$
\frac{2}{N+1}\left(A_{1}+\cdots+A_{N}\right)<q_{2}^{c}
$$

Now if $i$ of the $A_{k}$ take the value $q_{2}^{c}$ and the rest $N-i$ take the value $q_{1}^{c}$, inequality (20) says

$$
\frac{2}{N+1}\left((N-i) q_{1}^{c}+i q_{2}^{c}\right)<q_{2}^{c}
$$

which, dividing by $q_{2}^{c}$ and using $\lambda=q_{1}^{c} / q_{2}^{c}$, becomes

$$
\frac{2}{N+1}((N-i) \lambda+i)<1 \text {. }
$$

As we are assuming $\lambda>1 / 2$, this last equation implies

$$
2\left((N-i) \frac{1}{2}+i\right)<N+1 \quad \Longleftrightarrow \quad i<1 .
$$

\footnotetext{
${ }^{5}$ The solution can be easily found adding up both sides of all the equations, $(N+$ 1) $\left(q_{1}+\cdots+q_{N}\right)=2\left(A_{1}+\cdots+A_{N}\right)$, which tells us that $\sum_{j}^{\prime} q_{k}=2\left(A_{1}+\cdots+A_{N}\right)-q_{j}$. Replacing this value in equation $j$ of our system we get the solution given.
} 
Contradiction that proves that $A_{1}=A_{2}=\cdots=A_{N}=q_{1}^{c}$ and equation (20) becomes $\lambda<(N+1) /(2 N)$. The restrictions imposed on $\lambda$ are now

$$
\frac{1}{2}<\lambda<\frac{N+1}{2 N},
$$

which, in the case $N=2$ coincide with the known ones for duopoly.

Now, it is easy to see that, as $1 / 2<\lambda<(N+1) /(2 N)$, from the $2^{N}$ Cournot points in (19), only $N+2$ have all the coordinates positive:

$$
\begin{aligned}
\mathbf{I}_{\mathbf{0}} & =\left(\frac{2}{N+1} q_{1}^{c}, \ldots, \frac{2}{N+1} q_{1}^{c}\right) \\
\mathbf{I}_{\mathbf{N}+\mathbf{1}} & =\left(\frac{2}{N+1} q_{2}^{c}, \ldots, \frac{2}{N+1} q_{2}^{c}\right)
\end{aligned}
$$

and, for $j=1,2, \ldots, N$

$$
\mathbf{I}_{\mathbf{j}}=(\frac{2}{N+1}\left(2 q_{1}^{c}-q_{2}^{c}\right), \ldots, \underbrace{\frac{2 N}{N+1} q_{2}^{c}-\frac{2(N-1)}{N+1} q_{1}^{c}}_{j}, \ldots, \frac{2}{N+1}\left(2 q_{1}^{c}-q_{2}^{c}\right)) .
$$

\subsection{Existence and uniqueness of $I_{0}$}

Let us study what conditions need to be imposed on $q_{h}$ to guarantee the uniqueness of $\mathbf{I}_{\mathbf{0}}$. By the definition of $F_{j},(18), \mathbf{I}_{\mathbf{j}}$ can exist if

$$
0 \leq \sum_{j}^{\prime} q_{k} \leq q_{h},
$$

as $\mathbf{I}_{\mathbf{j}}$ is the intersection of

$$
F_{j}=q_{2}^{c}-\frac{1}{2} \sum_{j}^{\prime} q_{k}
$$

and all the other $F_{i}$ with $i \neq j$

$$
F_{i}=q_{1}^{c}-\frac{1}{2} \sum_{i}^{\prime} q_{k} .
$$

Thus if $0<q_{h}<\sum_{j}^{\prime} q_{k}$, when, for all $k, k \neq j q_{k}=2 /(N+1)\left(2 q_{1}^{c}-q_{2}^{c}\right), \mathbf{I}_{\mathbf{j}}$ will not exist. The condition on $q_{h}$ becomes

$$
0<q_{h}<\frac{2(N-1)}{(N+1)}\left(2 q_{1}^{c}-q_{2}^{c}\right) .
$$


From the fact that $(N-1) /(N+1)$ increases with $N$, the inequality will be verified if it is only checked for $N=2$,

$$
0<q_{h}<\frac{2}{3}\left(2 q_{1}^{c}-q_{2}^{c}\right)
$$

precisely the assumption made under duopoly.

In this way, the existence and uniqueness of $\mathbf{I}_{\mathbf{0}}$ for any $N$-poly, $N \geq 2$ is guaranteed if

$$
\frac{1}{6}(5-\lambda)<\mu<\frac{1}{2}(1+\lambda)
$$

our Assumption 5.

\subsection{Positive profit}

Finally, if our model has to have some feasibility we need to ensure that the $N$ firms in the $N$-poly equilibrium at $\mathbf{I}_{\mathbf{0}}$ make positive profit: for all $j, j=1, \ldots, N, \Pi_{j}\left(\mathbf{I}_{\mathbf{0}}\right)>0$. This is equivalent to

$$
c_{1}<\frac{4 b}{(N+1)^{2}}\left(q_{1}^{c}\right)^{2} .
$$

\subsection{Summing up}

We can summarize our findings in the case of $N$-poly for $N \geq 2$. The assumptions

$$
\left\{\begin{array}{rcc}
\frac{1}{2} & <\lambda< & \frac{N+1}{2 N} \\
\max \left(\lambda, \frac{1}{6}(5-\lambda)\right) & <\mu< & \frac{1}{2}(1+\lambda) \\
c_{1}<\frac{\left(a-d_{1}\right)^{2}}{(N+1)^{2} b}= & \frac{4 b}{(N+1)^{2}}\left(q_{1}^{c}\right)^{2}
\end{array}\right.
$$

guarantee the existence and uniqueness of a Cournot equilibrium point, $\mathbf{I}_{\mathbf{0}}$, with a non-quasi-competitive price structure $p_{1}>p_{N}$.

\subsection{The stability of the Cournot points}

Let us reorder the firms according to their speeds of adjustment and consider that $k_{1} \leq k_{2} \leq \cdots \leq k_{N}$. As before, the system of $N$ differential equations is

$$
\left\{\dot{q}_{i}=k_{i}\left(A_{i}-\frac{1}{2} q_{1}-\cdots-q_{i}-\cdots-\frac{1}{2} q_{N}\right) \quad i=1, \cdots, N .\right.
$$


Its solutions are given by the vector $\mathbf{q}(t)$ :

$$
q_{i}(t)=\sum_{j=1}^{N} D_{i, j} e^{-\alpha_{j} t}+\bar{q}_{i} \quad i=1, \cdots, N,
$$

where $\bar{q}_{i}$ are the different Cournot points (19). The $-\alpha_{j}$ are the eigenvalues of the matrix of the system,

$$
\left(\begin{array}{cccc}
-k_{1} & -k_{1} / 2 & \cdots & -k_{1} / 2 \\
-k_{2} / 2 & -k_{2} & \cdots & -k_{2} / 2 \\
\cdots & \cdots & \cdots & \cdots \\
-k_{N} / 2 & -k_{N} / 2 & \cdots & -k_{N}
\end{array}\right)=-\frac{1}{2}\left(\begin{array}{cccc}
2 k_{1} & k_{1} & \cdots & k_{1} \\
k_{2} & 2 k_{2} & \cdots & k_{2} \\
\cdots & \cdots & \cdots & \cdots \\
k_{N} & k_{n} & \cdots & 2 k_{N}
\end{array}\right)
$$

Now, the eigenvalues of the last matrix above are all real and positive. This can be seen just considering the characteristic polynomial,

$$
P(x)=\left|\begin{array}{cccc}
2 k_{1}-x & k_{1} & \cdots & k_{1} \\
k_{2} & 2 k_{2}-x & \cdots & k_{2} \\
\cdots & \cdots & \cdots & \cdots \\
k_{N} & k_{N} & \cdots & 2 k_{N}-x
\end{array}\right|
$$

and noticing that $P(0)=(N+1) k_{1} k_{2} \cdots k_{N}>0$ and

$$
P\left(k_{j}\right)=\left|\begin{array}{cccc}
2 k_{1}-k_{j} & k_{1} & \cdots & k_{1} \\
k_{2} & 2 k_{2}-k_{j} & \cdots & k_{2} \\
\cdots & \cdots & \cdots & \cdots \\
k_{N} & k_{N} & \cdots & 2 k_{N}-k_{j}
\end{array}\right|=k_{j} \prod_{i \neq j}\left(k_{i}-k_{j}\right) .
$$

If $k_{1}<k_{2}<\cdots<k_{N}$, we have that the signs of the sequence $P\left(k_{1}\right), P\left(k_{2}\right), \ldots$, $P\left(k_{N}\right), P(\infty)$ are alternated This fact guarantees that the $N$ roots of $P(x)$ are one in each interval $\left(k_{1}, k_{2}\right), \ldots,\left(k_{N-1}, k_{N}\right),\left(k_{N}, \infty\right)$. If $\ell$ consecutive $k_{i}$ 's are equal, $k_{i}$ itself becomes a root of multiplicity $\ell-1$, which can be seen differentiating $\ell$ times $P(x)$ from its determinant form, (22) and the rest of the roots remain in the same intervals as before.

Consequently, the $\alpha_{j}>0$ for $j=1, \ldots, N$. This proves that the stationary solutions of our system (or systems) are globally stable.

\section{$4 \quad$ Stability for a new entry}

It is not easy to carry out the study of the stability of the model following the same steps as in duopoly. The complexity of the regions in which $[0, a / b]^{N}$ gets divided makes it much too difficult. We have two ways to try to establish 
it: to apply a test as Hahn's test, see Hahn (1962), which is clear-cut and easy to apply; or to move carefully from the $N$-poly equilibrium situation to the new situation with a new entry. The first way, requires the differentiability of our cost function which can only be ensured if the actual outputs of all firms keep on the same side of $q_{m}$. Let us explore the second, more direct, alternative. Let us suppose then that our $N$-poly firms' output is at the Cournot point $\left(2 q_{1}^{c} /(N+1), \ldots, 2 q_{1}^{c} /(N+1)\right)$, and the new firm enters with an output $q_{N+1}(0)$ in the range $\left[0,2 q_{1}^{c} /(N+1)\right]$. Let us prove that with these initial conditions, the new system has as its new equilibrium point

$$
\mathbf{q}\left(q_{1}, \ldots, q_{N+1}\right)=\left(\frac{2}{N+2} q_{1}^{c}, \cdots, \frac{2}{N+2} q_{1}^{c}\right) .
$$

We will prove it by showing that with the initial conditions just mentioned, the orbits of the motion are confined in the region where the prevailing system of differential equations is always the same; that is the one for which we consider the reaction function in (18) corresponding to $\sum_{j}^{\prime} q_{k}>q_{h}(j=$ $1 . . N+1)$. If $Q(t)$ denotes the total output of the industry at moment $t$,

$$
\left\{\dot{q}_{i}=k_{i}\left(q_{1}^{c}-\frac{1}{2} Q-\frac{1}{2} q_{i}\right) \quad(i=1, \cdots, N+1) .\right.
$$

Adding up all the equations before, we set up a differential equation that $Q(t)$ must verify:

$$
\dot{Q}=\sum_{i=1}^{N+1} k_{i}\left(q_{1}^{c}-\frac{1}{2} Q-\frac{\sum_{i=1}^{N+1}\left(k_{i} q_{i}\right)}{2 \sum_{i=1}^{N+1} k_{i}}\right) .
$$

Now, turning to our purpose, we want to make sure that the orbit of vector $\mathbf{q}(t)$ verifies for all $t, \sum_{i}^{\prime} q_{j}(t)>q_{h} \quad(i=1, \ldots, N+1)$.

The initial conditions we have, $\left(2 q_{1}^{c} /(N+1), \ldots, 2 q_{1}^{c} /(N+1), q_{N+1}(0)\right)$ verify

$$
\left\{\begin{array}{l}
0 \leq q_{i}(0) \leq \frac{2}{N+1} q_{1}^{c} \quad(i=1, \ldots, N+1) \\
\frac{2 N}{N+1} q_{1}^{c} \leq Q(0) \leq 2 q_{1}^{c} .
\end{array}\right.
$$

Let us see that the orbit of $\left(q_{1}(t), \ldots, q_{N+1}(t)\right)$ and also that of $Q(t)$, get confined to that same region, not only for the initial values but for all $t$.

Lemma 1 As long as for $t>0$ and for all $i=1,2, \ldots, N+1$, we have $0 \leq q_{i}(t) \leq 2 q_{1}^{c} /(N+1)$ then we will have

$$
\frac{2 N}{N+1} q_{1}^{c}<Q(t)<2 q_{1}^{c}
$$


Proof To prove this result we will use a simple straightforward mathematical fact. If $f:[0, \infty) \longrightarrow \mathbb{R}$ is a continuously differentiable function and $f(0)<H$, then either $f(t)<H$ forever or the first time that $f$ reaches the value $H$, let us say for $t=t^{*}>0$, we must have $f^{\prime}\left(t^{*}\right) \geq 0$. If $f(0)>H$, then either $f(t)>H$ always or the first time that $f\left(t^{*}\right)=H$ we must have $f^{\prime}\left(t^{*}\right) \leq 0$. Lastly if $f(0)=H$, if $f^{\prime}(0)>0$, then either $f(t)>H$ for all $t>0$ or the first time after $t=0$ that the value $H$ is attained, we must have $f^{\prime}\left(t^{*}\right) \leq 0$ and if $f^{\prime}(0)<0$, then either $f(t)<H$ for all $t>0$ or the first time after 0 we have $f\left(t^{*}\right)=H$ we must have $f^{\prime}\left(t^{*}\right) \geq 0 .{ }^{6}$

With this result in mind, and noticing that all our $q_{i}$ are continuously differentiable infinitely many times, and consequently $Q$ too, we reason in the following way.

Under the hypothesis of the lemma, we have $Q(t)=\sum q_{i}(t) \leq 2 q_{1}^{c}$. The equal sign can only be if all the $q_{i}$ 's take the value $2 q_{1}^{c} /(N+1)$ at the same time. If $t^{*}>0$ is the first time this happens, we will have for all $i=1,2, \ldots, N+1 \dot{q}_{i}\left(t^{*}\right)=k_{i}\left(-2 q_{1}^{c} /(N+1)<0\right.$ and consequently, $\dot{Q}\left(t^{*}\right)=\sum \dot{q}_{i}(t)<0$. As $Q(0) \leq 2 q_{1}^{c}$ (and if $Q(0)=2 q_{1}^{c}, \dot{Q}(0)<0$ ) by the mathematical remark we should have $\dot{Q}\left(t^{*}\right) \geq 0$, contradiction that proves that for all $t>0, Q(t)<2 q_{1}^{c}$.

If $t^{*},\left(t^{*}>0\right)$ is the first time that $Q\left(t^{*}\right)=2 N q_{1}^{c} /(N+1)$, then for all $i=1, \ldots, N+1$ we have $\dot{q}_{i}\left(t^{*}\right) \geq 0$ which implies that $\dot{Q}\left(t^{*}\right) \geq 0$. Equality is in fact impossible as it would imply that all $\dot{q}_{i}\left(t^{*}\right)=0$ simultaneously and this can only happen if all the $q_{i}\left(t^{*}\right)=2 q_{1}^{c} /(N+1)$ which would mean that $Q\left(t^{*}\right)=2 q_{1}^{c}$, which is absurd. We have then $\dot{Q}\left(t^{*}\right)>0$. Now, $Q(0) \geq$ $2 N q_{1}^{c} /(N+1)$ (and if $Q(0)=2 N q_{1}^{c} /(N+1)$, then $\dot{Q}(0)>0$ ), thus by our mathematical remark we should have $\dot{Q}\left(t^{*}\right) \leq 0$. This contradiction proves that for all $t>0, Q(t)>2 N q_{1}^{c} /(N+1)$.

Q.E.D.

Now we must see that, for all $t$, the hypothesis of our lemma is valid, that is to say $0 \leq q_{i}(t) \leq 2 q_{1}^{c} /(N+1)$.We will reason as in the proof of the lemma. If $t^{*}>0$ is the first moment in time that one of the firms $q_{\ell}\left(t^{*}\right)=0$, the lemma applies for $0 \leq t \leq t^{*}$ and we can infer that $Q\left(t^{*}\right)<2 q_{1}^{c}$ which implies $q_{1}^{c}-(1 / 2) Q\left(t^{*}\right)>0$ and this means $\dot{q}_{\ell}\left(t^{*}\right)>0$. Using our mathematical result we should have $\dot{q}_{\ell}\left(t^{*}\right) \leq 0$. In this way we see that $q_{\ell}(t)$ cannot be 0 for any value of $t$. An analogous reasoning leads us to the result that $q_{\ell}\left(t^{*}\right)$ cannot reach $2 q_{1}^{c} /(N+1)$ ever.

\footnotetext{
${ }^{6}$ If $f(0)=H$ and $f^{\prime}(0)=0$ then there might not be a FIRST time after $t=0$ where $f(t)=H . f$ can cross $y=H$ an infinity of times. Think of $f(x)=x^{3} \sin (1 / x) ; f(0)=0$.
} 
Summing up, we have that

$$
\left\{\begin{array}{l}
0<q_{i}(t)<\frac{2}{N+1} q_{1}^{c} \\
\frac{2 N}{N+1} q_{1}^{c}<Q(t)<2 q_{1}^{c} .
\end{array} \quad \text { for all } t>0 .\right.
$$

These results prove that, for all $i$ the orbit of $q_{i}(t)$ remains confined to the region where $\sum_{i}^{\prime} q_{j}>q_{h}$ :

$$
\sum_{i}^{\prime} q_{j}(t)=Q(t)-q_{i}(t)>\frac{2 N}{N+1} q_{1}^{c}-\frac{2}{N+1} q_{1}^{c}=\frac{2(N-1)}{(N+1)} q_{1}^{c}>q_{h} .
$$

Notice that for $N=1$ this last chain of inequalities says simply that $q_{1}>0$ or $q_{2}>0$ and we cannot conclude that our set of differential equations is always the same. That explains the different treatment for the case of monopoly to duopoly.

Incidentally, the fact that for all $i$, and all $t, q_{i}(t)<2 q_{1}^{c} /(N+1)<q_{m}$ allows us to use Hahn's condition (see Hahn (1962)) to prove stability: $d^{\prime}<$ $C^{\prime \prime}$ when $d^{\prime \prime}=0$ and $C^{\prime \prime}=$ constant.

\section{A numerical example}

We finish by exhibiting a numerical example of our model. To build it we need to go from one assumption to another taking good care that no contradictions arise.

Let our demand function be $p=100-2 q$, and $N=5$. The cost function of our 5 firms has to be carefully chosen:

1. As $a=100$ and $b=2$ we must have $q_{2}^{c}<25$. Let us choose $q_{2}^{c}=24$.

2. As $N=5,1 / 2<\lambda<(5+1) /(2 \times 5)$, we choose $\lambda=0.55$.

3. Hence $q_{1}^{c}=\lambda q_{2}^{c}=13.2$.

4. The parameter $\mu$ must satisfy

$$
0.7416666 \ldots=\max (\lambda,(1 / 6)(5-\lambda))<\mu<(1 / 2)(1+\lambda)=0.775 \text {, }
$$

we choose $\mu=0.75$.

5. Thus $q_{m}=\mu q_{2}^{c}=18$.

6. From $d_{i}=a-2 b q_{i}^{c}(i=1,2)$, we have $d_{1}=47.2 ; d_{2}=4$. 
7. The fixed costs $c_{1}$ must verify $c_{1}<(8 / 36) \times 13.2^{2}$. We choose $c_{1}=10$.

8. Lastly from $c_{2}=c_{1}+\left(d_{1}-d_{2}\right) q_{m}$ we have $c_{2}=787.6$.

The cost function of our example will be:

$$
C(q)= \begin{cases}10+47.2 q & \text { if } 0 \leq q<18 \\ 787.6+4 q & \text { if } 18 \leq q<50 .\end{cases}
$$

Table 1 shows the different total outputs and prices as we move from $N=1$ to $N=5$ :

TABLE 1

OUTPUTS AND PRICES

\begin{tabular}{ccc}
\hline \hline$N$ & $Q$ & $P_{N}$ \\
\hline 1 & 24.00 & 52.00 \\
2 & 17.60 & 64.80 \\
3 & 19.80 & 60.40 \\
4 & 21.12 & 57.76 \\
5 & 22.00 & 56.00 \\
\hline
\end{tabular}

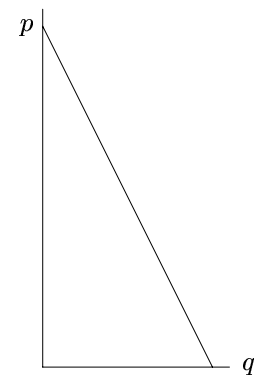

Demand

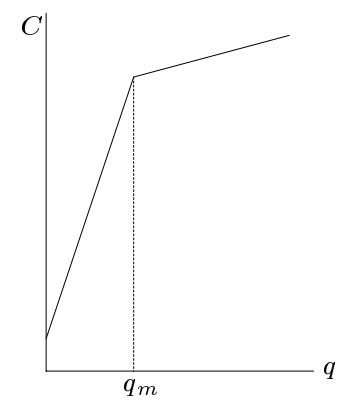

Cost

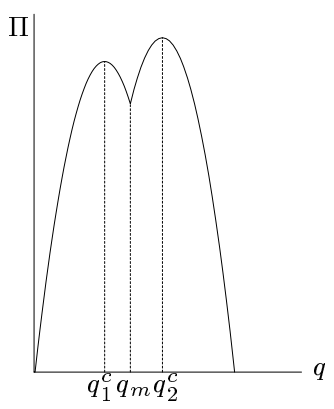

Monopoly profit

Figure 4

\section{Conclusions}

We have exhibited a classical Cournot oligopoly model in which monopoly price is lower than any oligopoly price for successive free entry up to $N$ firms with $N$ given. In all cases the equilibrium solution reached is unique and stable under habitual adjustment mechanisms. We stress the fact that in our model, the choosing of one of the parameters $\lambda$ is confined to a small interval, $1 / 2<\lambda<(N+1)) /(2 N)$. When $N \rightarrow \infty, \lambda \rightarrow 1 / 2,2 q_{1}^{c} \rightarrow q_{2}^{c}$ 
and then the oligopoly solution tends to a total industry output of $2 q_{1}^{c}$ as $\lim 2 N q_{1}^{c} /(N+1)=2 q_{1}^{c}$. In this case, it is easy to see that our marginal cost is exactly the market price and we are in perfect competition with a market output and price which is exactly the same as in monopoly.

\section{References}

Daughety, A. F., ed. (1988): Cournot Oligopoly: Characterization and Applications. Cambridge: Cambridge University Press.

Fisher, F. (1961): "The stability of the cournot oligopoly solution: The effects of speeds of adjustment and increasing marginal costs". Review of Economic Studies, XXVII (2), 125-135.

Frank JR., C. AND QuANDT, R. (1963): "On the existence of cournot equilibrium". International Economic Review, 4, 92-96.

Hahn, F. (1962): "The stability of the cournot oligopoly solution". Review of Economic Studies, XXIX, 329-331.

McManus, M. (1962): "Numbers and size in cournot oligopoly". Yorkshire Bulletin of Economic and Social Research, 14, 14-22.

- (1964): "Equilibrium, numbers and size in cournot oligopoly". Yorkshire Bulletin of Economic and Social Research, 16, 68-75.

Okuguchi, K. (1974): "Quasi-competitiveness and cournot oligopoly". Review of Economic Studies, XL, 145-148.

- (1976): Expectations and Stability in Oligopoly Models, vol. 138 of Lecture Notes in Economics and Mathematical Systems. Berlin, Heidelberg, New York: Springer-Verlag.

Okuguchi, K. And Suzumura, K. (1971): "Uniqueness of the cournot oligopoly equilibrium: A note". Economic Studies Quarterly, 22, 81-83.

QuAnDT, R. (1967): "On the stability of price adjusting oligopoly". The Southern Economic Journal, XXXIII (3), 332-336.

Rosenthal, R. (1980): "A model in which an increase in the number of sellers leads to a higher price". Econometrica, 48 (6), 1575-1579.

Ruffin, R. (1971): "Cournot oligopoly and competitive behaviour". Review of Economic Studies, XXXVIII, 493-502. 
Salop, S. (1979): "Monopolistic competition with outside goods". Bell Journal of Economics, 10, 141-156.

Satterthwaite, M. (1979): "Consumer information, equilibrium industry price, and the number of sellers". Bell Journal of Economics, 10, 483-502. 